\title{
Loss of vision from pituitary tumours causing chiasmal compression
}

\author{
Michael Powell
}

Received: 3 September 2010 /Accepted: 7 September 2010/Published online: 29 September 2010

(C) Springer-Verlag 2010

Surgery for visual loss caused by chiasmal compression from pituitary adenomas has a long history in neurosurgery. Even in the pre-microspcope era, craniotomies, as described by the pioneers of our specialty, although risky could be very successful in restoring vision, and once microsurgery was established, outcomes steadily improved.

Good outcomes for vision have been reported for a long time. Less than a quarter of a century ago, when most of these procedures were done as craniotomies and transsphenoidal surgery was considered dangerously 'avant garde' in traditional circles, visual outcomes as reported by Symon in 1979 [1] in his large craniotomy series could be exceptional and have never been improved on by 'transsphenoidalists'. It must be noted that his series excluded larger macroadenomas, and the so called 'giant' tumours reported separately did less well.

Since the general acceptance of transsphenoidal microsurgery for these tumours, there have been many reports on visual outcome for visual loss from adenomas. Although many are flawed in statistical terms, as they treat each eye as a separate entity when in fact they are pairs; however, there can be no doubt that surgery is extremely effective in restoring vision in the majority of cases, with about half returning to normal eye sight.

Most series report fields and acuity outcomes. There is also an inherent technical statistical problem in analysis concerning field assessment. Field losses are graded by qualitative methods which do not easily lend themselves to statistical analysis. This is made even more difficult as the

\section{Powell $(\square)$}

The National Hospital for Neurology and Neurosurgery,

Queen Sq,

WC1N 3BG London, UK

e-mail: michael.powell@uclh.nhs.uk field assessment methods used (Goldmans, Humphreys etc.) differ not only within individual series but of course, between different series, making interseries comparison to some extent meaningless. It seems little understood that acuity changes usually reflect the later stages of chiasmal compression as detail vision represented by acuity is mainly represented in the retina at the macular. Macular loss is a late phenomenon because of the protective disposition of the macular fibres in the chiasm, thus small changes in acuity are probably irrelevant although statistically, there may appear to be slight worsening.

Visual field and acuity worsening in all series are relatively low, usually in the $2-5 \%$ range, and mortality even less. The main risks from surgery are from damage to the normal gland function (which is easily replaced), and from CSF leaks.

The take-home message is that the risk of transsphenoidal surgery is very low. As is well known, these risks lessen with experience, although, interestingly, visual outcome does not seem to improve.

The evidence for that comes from the much quoted paper from Cohen [2], in which a large series of adenomas is reported. Visual improvement for fields is still around $80 \%$, very much par for the course. Interestingly, the number of surgeons for the number of patients is high, so over the study period it is certain that numbers are included from surgeons with relatively little experience, unlike the single surgeon series reported from 'masters' such as Laws, Teasdale, etc.

It is also of interest that with improvements in modern imaging, and with the introduction of endoscopic techniques, there is no further improvement in visual outcome.

What is left for outcome series to report? It would certainly be interesting to try to tie visual outcome to length of history. It seems intuitive that the longer the compression, the worse 
the outcome, especially as we know that in patients who do not have surgery and with known compression, vision usually gets worse with time. Unfortunately, the link is difficult to prove, perhaps because there are always a number of patients who cope with their visual loss for quite some time, and present late with severe vision deficits. In a few words, short histories, bad vision, poor outcome.

The paper published in this edition [3] is part of a twopaper series, the other part being published elsewhere relating to analysis. It seeks to compare patients with and without visual loss, who have pituitary tumour surgery. The outcomes and conclusions reported are a little disappointing for such a large piece of meticulous work. Perhaps there is not much left to say, although the authors do claim a couple of minor original points from their series, however.

Their main point, the comparison with a normal vision group with pituitary surgery, is of course novel, although seems to add nothing that would not be expected.

Perhaps it is reassuring to know that one's patients' chance of retaining vision, if not previously affected, is good. All their patients, starting with normal vision, retained it unchanged. However, the series does include $3 \mathrm{~mm}$ microadenomas and with an average of $14 \mathrm{~mm}$, a size, seems hardly surprising that it remains unchanged and the patients not at risk. Unfortunately, we still do not know what the risk is for those patients who undergo surgery with tumours causing chiasmal compression but without visual loss, these days a relatively frequent occurrence. The paper could be interpreted to infer that visual loss is not a risk.
The authors also debate the failure of complete recovery of vision compared to those patients without preoperative visual loss. They speculate that this may be because of concomitant eye disease, influencing recovery. In our experience, concomitant eye disease delays the diagnosis of chiasmal compression, usually whilst the ophthalmologist manages the much more common conditions of cataracts and glaucoma. The average delay is for 612 months. It is also notable that chiasmal compression in experimental animals leads to significant demyelination, so it is of no surprise that our patients do not necessarily return to normal. Perhaps we should simply be grateful that they do recover at all.

Conflicts of interest None.

\section{References}

1. Symon L, Jakubowski J (1979) Transcranial management of pituitary tumours with suprasellar extension. J Neurol Neurosurg Psychiatry 42:123-33

2. Cohen AR, Cooper PR, Kupersmith MJ, Flamm ES, Ransonhoff J (1985) Visual recovery after transsphenoidal removal of pituitary adenomas. Neurosurgery 17:446-452

3. Kristof RA, Kirchhofer D, Handzel D, Neuloh G, Schramm J, Mueller CA, Eter N (2010) Pre-existing chiasma syndromes do not entirely remit following transsphenoidal surgery for pituitary adenomas. Acta Neuochir (in press) 